\title{
Revelations About Carotid Body Function Through its Pathological Role in Resistant Hypertension
}

\author{
Julian F. R. Paton • Laura Ratcliffe • Dagmara Hering • \\ Jacek Wolf • Paul A. Sobotka • Krzysztof Narkiewicz \\ Published online: 5 July 2013 \\ (C) The Author(s) 2013. This article is published with open access at Springerlink.com
}

\begin{abstract}
Much recent attention has been given to the carotid body because of its potential role in cardiovascular disease states. One disease, neurogenic hypertension, characterised by excessive sympathetic activity, appears dependent on carotid body activity that may or may not be accompanied by sleep-disordered breathing. Herein, we review recent literature suggesting that the carotid body acquires tonicity in hypertension. We predict that carotid glomectomy will be a powerful way to temper excessive sympathetic discharge in diseases such as hypertension. We propose a model to explain that signalling from the 'hypertensive' carotid body is tonic, and hypothesise that there will be a sub-population of glomus cells that channel separately into reflex pathways controlling sympathetic motor outflows.
\end{abstract}

Keywords Peripheral arterial chemoreceptor · Sympathetic nervous system $\cdot$ Hypertension $\cdot$ Central integration $\cdot$ Carotid body resection $\cdot$ Chemoreceptor reflex sensitivity

\section{Introduction}

The aetiology of many modern-day diseases, such as the metabolic syndrome, is often associated with alterations in the autonomic nervous system. The relatively sudden

J. F. R. Paton $(\bowtie) \cdot$ L. Ratcliffe

School of Physiology \& Pharmacology, Bristol Heart Institute,

University of Bristol, Medical Sciences Building, Bristol BS8 1TD

England, UK

e-mail: Julian.F.R.Paton@Bristol.ac.uk

D. Hering $\cdot J$. Wolf $\cdot$ K. Narkiewicz

Department of Hypertension and Diabetology, Medical University

of Gdansk, Debinki 7c, 80-952 Gdansk, Poland

P. A. Sobotka

Cibiem NC1., 134 W 26th St., Suite 1011, New York, NY 10001, USA acceptance that autonomic imbalance is both causative and essential for the maintenance of disease states in animal models and humans, has led to an eruption of interventional approaches to manage these diseases. Modulation of the autonomic nervous system, whether via stimulation $[1,2]$ or ablation of organ-specific afferents and denervation of sympathetic post-ganglionic fibres targeting selected vascular beds [3•], is a strong current trend producing spectacular results. Here, we consider an intervention that is based on pre-clinical proof of principle data and previously published clinical results supporting carotid body modulation as a treatment for neurogenic hypertension.

\section{The Problem of Hypertension is that Not Only Does It Not Go Away, It Gets Worse}

Carey has recently proposed that $14 \%$ of the world's treated hypertensive patients are resistant to contemporary pharmacological therapy [4]. Even if this number is an overestimate, we are still left with a substantial clinical problem, given that there are presently almost 1 billion hypertensives worldwide [5]. Most hypertensive patients are well managed with antihypertensive drugs, yet there appears to be an increasing trend to greater resistance [4]. As Carey reports, resistant hypertensives have increased from $5.5 \%$ in $1988-1994$, to $8.5 \%$ in 1999-2004 and $11.8 \%$ in 2005-2008 [4]. A resistant hypertensive is a patient with office blood pressure $>140 / 90 \mathrm{mmHg}$, despite taking $\geq 3$ anti-hypertensive medications, ideally including a diuretic [6]. However, we believe the clinical problem of blood pressure management extends beyond drug resistance, and includes hypertensive patients that are drug intolerant due to adverse effects impacting on quality of life, often a consequence of high drug dosing and multi-pill therapy. If accepted that the sympathetic nervous system is in part responsible in the etiology and maintenance of hypertension $[7-9,10 \bullet, 11,12]$, then we need to find innovative ways to 
control it. Although highly effective, the early attempts at gross surgical $[13,14]$ and then pharmacological sympathectomy [15] are unsurprisingly poorly tolerated. Given that sympathetic nervous system function is controlled differentially the idea of targeted sympathectomy, such as to the kidney [3•, 17•] or splanchnic vascular bed [16], has had considerable success in lowering blood pressure clinically and in animal models. However, the majority of patients receiving renal denervation have remained on anti-hypertensive medication and hypertensive $[3 \bullet, 17 \bullet]$. Given the hypothetical aim of curing hypertension and reducing/removing antihypertensive medication, especially those with severe life quality impeding side effects, further exploration into novel interventional therapies is warranted. We will discuss the idea of removing a distinct sympatho-excitatory afferent drive as a novel treatment for neurogenic hypertension.

\section{The Peripheral Chemoreceptor}

The peripheral or arterial chemoreceptors located in the carotid body send signals via a branch of the carotid sinus nerve to the nucleus of the solitary tract located in the dorsomedial medulla [18]. The carotid body consists of supporting cells (or type II cells, analogous to astrocytes) and glomus cells (type I cells) that embryologically originate from neural crest cells. Glomus cells are morphologically distinct as large and spherical in shape and are highly sensitive to oxygen delivery, arterial oxygen and carbon dioxide levels, blood $\mathrm{pH}$, reduced blood flow (e.g. hemorrhage), inorganic phosphate and sodium cyanide [19]; the latter being used as a convenient experimental tool to produce tissue hypoxia and profound stimulation [20]. The carotid body is profusely perfused with blood [21] and the arterioles are innervated by the sympathetic nervous system. This is an important point as excessive sympathetic activity could potentially trigger activation of the chemoreceptor (via hypoperfusion). Persistent sympathetic activity might also re-model carotid body arterioles causing alterations in perfusion that could directly affect sensitivity of the chemoreceptor. The carotid body cells produce a vast array of chemical transmitters (including dopamine, noradrenaline, substance P) that are released to trigger a receptor potential on nearby afferent endings, resulting in their depolarisation and action potential generation [22]. In some species, such as rabbit, cat and human, there are additional glomus tissue accumulations on the aorta and subclavian arteries [23]. Aortic chemoreceptors have a distinct reflex response [23] compared to those evoked from carotid chemoreceptors (see below), and for this reason our focus will be on the carotid body.

\section{Carotid Body Reflex Response Patterns and the Intimate Association with Respiration}

Stimulation of the carotid body chemoreceptors triggers hyperventilation, an important protective reflex mechanism ensuring blood gas homeostasis. The peripheral chemoreceptors may also contribute to dyspnoea [24] and arousal [25], which is likely due to their suprabulbar projections [26].

The reflex cardiovascular response evoked from the carotid body is interesting, as it comprises a primary and secondary response $[27,28]$. The pattern of response evoked is dependent on the degree to which respiration increases. Hyperventilation recruits into play an afferent signal originating from mechanoreceptors surrounding bronchioles that on inflation trigger the pulmonary stretch receptor (HeringBreuer) reflex giving the secondary response. This secondary response consists of tachycardia and vasodilatation; the former is mediated by cardiac vagal withdrawal and increased cardiac sympathetic tone, and the latter via inhibition of sympathetic vasomotor tone. This competes with the primary response of profound bradycardia (vagally mediated) and sympathetically mediated vasoconstriction. The resultant response depends on the relative strength of the two afferent reflexes and the magnitude of the respiratory response, which is dependent on animal species. The primary response prevails in the following examples: a diving mammal; human face immersion into cold water; sleep apnea; sudden infant death syndrome; and in anesthetised and mechanically ventilated animals. The bradycardia is potent, abolished with atropine and most likely protective in function to reduce cardiac metabolism and preserve oxygen. Intriguingly, despite the potent bradycardia, the sympathetic nervous system targeting the heart is co-activated [29] along with the cardiac vagal system and may increase cardiac force of contraction.

The type of stimulus used to activate the peripheral chemoreceptors will also dictate the response pattern. Using systemic hypoxia, for example, will produce a direct hyperpolarising effect on the vascular smooth muscle causing blood pressure to fall. This will unload baroreceptors that may trigger a compensatory response, although it is acknowledged that their interaction with peripheral chemoreceptors is antagonistic [20,30,31]. Finally, the strength of the stimulus may also produce a graded response, perhaps overturning coincident reflexes originating from pulmonary receptors in spontaneously breathing animals. When the stimulus is intense, peripheral chemoreceptors can trigger an alerting response, including the classical behavioural and visceral adjustments associated with fight or flight [19, 25]. In the context of carotid chemoreceptors and hypertension, this is most relevant, as it has been hinted that continuous activation of the fight or flight response (so called defence response) could lead to systemic hypertension [32]. 


\section{The Association Between Peripheral Chemoreceptor Sensitivity and Sympathetic Nerve Activity Excess}

The peripheral chemoreceptor reflex sensitivity has been shown to be significantly enhanced in both patients with primary hypertension [33-37, 38• 39] and spontaneously hypertensive $(\mathrm{SH})$ rat $[40 \bullet \cdot$; this has included the ventilatory and sympathetic nerve activity response components. Incidentally, an increased hypoxic ventilatory response is characteristic of heart failure patients. This increased peripheral chemoreceptor sensitivity is a prognostic indicator for mortality in these patients [41].

Hypersensitivity of the carotid body occurs before hypertension is established in both SH rats [40••] and white coat hypertensive patients [36]. Repetitive exposure of conscious normotensive rats to hypoxia can induce mild hypertension [42]. This is dependent on stimulation of peripheral chemoreceptors, as the hypertension is abolished by a priori carotid body removal [42]. This model of chronic intermittent hypoxia has been used as a model for sleep apnea. The model is tenuous, because it exhibits hypocapnia (not hypercapnia) and increased ventilation (not apnea). However, the hypertension produced is caused by activation of the sympathetic nervous system in rats [43•]. This is consistent with the sympathetic activation associated with the hypertension seen during obstructive or central sleep apnea in human patients with or without heart failure $[44,45]$. Repeated peripheral chemoreceptor activation in sleep apnea has also been proposed to contribute to derangements in metabolism and obesity [46••]. Further, there is evidence that patients with sleep apnea develop tonic peripheral chemoreceptor afferent discharge that functionally drives muscle sympathetic activity [47]. Repeated carotid body activation in rats and humans results in enhanced respiratory-sympathetic coupling and increased muscle vasoconstrictor activity, which may contribute to the development of hypertension [10•, 33, 34, 36, 43•, 45]. Recently, we found that adrenomedullin in the rostral ventrolateral medulla was key for producing the long-term potentiation of sympathetic nerve activity evoked with repetitive stimulation of the carotid body (Colombari DS, Colombari E, Murphy D \& Paton JF- unpublished data). The mechanisms causing release of adrenomedullin are unknown, but intermittent hypoxia cannot be ruled out. It should also be recognised that rostral ventrolateral medullary neurons are themselves intrinsically excited by hypoxia, providing a 'fail safe' mechanism for driving sympathetic activity in conditions of prolonged apnea and severe hypoxia, for example [47].

\section{The 'Hypertensive' Carotid Body}

Morphological studies have revealed that carotid bodies are enlarged in hypertensive patients [48]. In the spontaneously hypertensive (SH) rat, the glomus cells are hypertrophied suggesting up regulation of protein synthesis and there is angiogenesis within the microvasculature relative to controls [49]. Above, we described that the peripheral chemoreceptor reflex is sensitised in patients with hypertension. The mechanism/s for this remain to be fully understood. However, in pre-hypertensive (juvenile) SH rats, type I cells display enhanced sensitivity to low $\mathrm{pH}$, due to increased expression of two acid-sensing non-voltage gated channels, ASIC3 and TASK1 [40••]. There is also evidence that the balance between carbon monoxide and hydrogen sulphide signalling within the $\mathrm{CB}$, as well as the relative activity of HIF $1 \alpha$ versus HIF $2 \alpha$ play a role [50]. Animal models of chronic heart failure have revealed that carotid body (CB) chemoreceptor activity is augmented by activation of angiotensin II receptors [51], impaired nitric oxide synthase activity [52], reduced CB blood flow [53•], enhanced adenosine monophosphate-activated protein kinase [54], and inflammation [55], but whether these changes are also seen in hypertensive carotid bodies remains to be elucidated. Heightened sympathetic drive to the arterioles of the CB may also contribute to its hyperactivity due to hypoperfusion (via vasoconstriction and vascular remodelling). Kato et al. described that there is significant dopamine $\beta$-hydroxylase (DBH) immunoreactivity in fibres around glomus cells [56॰]. A possibility is that the larger morphology of the $\mathrm{CB}$ in the $\mathrm{SH}$ rat may be the effect of the sympathetic innervation [57]. Interestingly, glomus cells in SH rats exhibited greater DBH immunoreactivity relative to normotensive rats, suggesting that this amine is released in abundance in hypertension [56•]. This is in stark contrast to the normal age-dependent (i.e. maturational) reduction in $\mathrm{DBH}$ in the healthy $\mathrm{CB}$ [57].

Given the role for the immune system in hypertension and the pro-inflammatory function of the sympathetic nervous system [58-60], inflammation within the carotid body may also be a potential mechanism. Activation of toll-like receptors increases NLRP3 inflammasome expression in carotid body cells, which enhances IL- $1 \beta$ production that acts in an autocrine manner to enhance peripheral chemoreceptor drive $[61 \bullet]$.

\section{Making the Distinction Between Peripheral Chemoreflex Sensitivity and Carotid Body Tonicity}

We previously raised the issue of whether reflex chemosensitivity is always associated with carotid body hyperactivity (tonicity), or if increased carotid body tone can occur in the absence of increased chemosensitivity [62]. This remains to be fully validated. Nevertheless, it may be crucial to understand the relationship between reflex sensitivity and tonicity if the carotid body is targeted therapeutically (see below). Hyperoxia has been used to demonstrate 


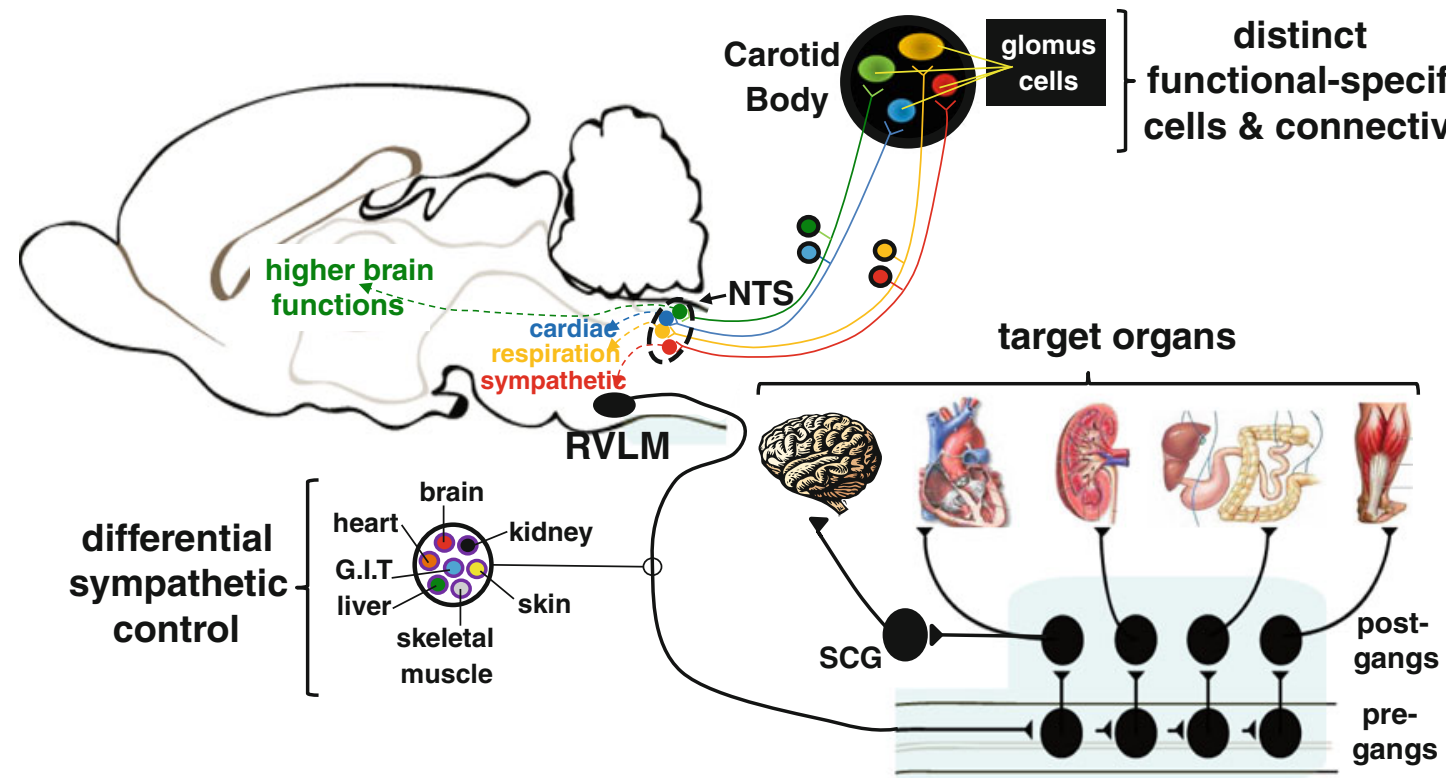

Fig. 1 Mechanistic, hypothetical model depicting separate lines of communication from individual glomus cells to distinct functional neuronal networks regulating a range of reflex visceral and behavioural responses. It is also conceivable that different glomus cells connect to

carotid body tonicity by inactivating aberrant discharge $[38 \bullet, 63]$. In SH rats, hyperoxia (100\% oxygen) was found to reduce arterial pressure and renal sympathetic nerve activity [64••]. In human hypertensive patients [38•] and patients with sleep apnea [46••], a similar effect was observed, including reductions in arterial pressure and muscle sympathetic nerve activity. The cause for this pathological tone is not known, but could include mechanisms described above, driving sensitivity. These mechanisms demonstrate a fundamental role of the carotid bodies in the pathogenesis of cardiovascular disease including essential hypertension.

\section{The Carotid Body Can Generate Hypertension: A Pre-Clinical Proof of Principle Study}

Our recent endeavours with a pre-clinical model of hypertension have shown that carotid body (CB) ablation can prevent both the development of hypertension and reduce established hypertension in the $\mathrm{SH}$ rat $[64 \bullet \bullet, 65]$. The effect is prompt (1-2 days post-surgery) and very well maintained (up to 6 weeks). It is associated with a profound reduction in sympathetic nerve activity ( $\sim 55 \%)$, improved baroreceptor reflex gain and renal function, and reduced systemic inflammation [64••]. The anti-hypertensive effect is independent of the renal nerves, yet provides a summative effect if performed after renal denervation [64••]. As reviewed recently [62], unilateral carotid body resection has been used extensively as a treatment for dyspnoea in asthmatic and different pre-motor sympathetic neurons in the rostral ventrolateral medulla (RVLM) controlling distinct target organs. Abbreviations: post-gangs post-ganglionic sympathetic neuron; pre-gangs pre-ganglionic sympathetic neurons

chronic obstructive pulmonary disease patients. In two studies, blood pressure changes were reported. Nakayama et al. documented sustained blood pressure reductions following $\mathrm{CB}$ resection [66]. They reported a reduction in systolic blood pressure from $170 \mathrm{mmHg}$ to $130 \mathrm{mmHg}$ at 5-days post-op that was maintained throughout the duration of the study (6 months). Additionally, Winter et al. noted acute blood pressure reductions after CB removal [67]. No clinical trial has studied the effect of unilateral carotid body resection for hypertension in humans.

\section{Novel Insights Revealed by the Hypertensive Carotid Body}

An understanding of the drivers of carotid body tonicity becomes crucial academically as well as clinically. We raise the question as to whether all glomus cells in hypertension become active or perhaps only those regulating sympathetic activity. Said differently, are there distinct connections from the carotid body into the nervous system targeting different reflex pathways (Fig. 1)? The observation that deactivation of carotid bodies in $\mathrm{SH}$ rats and humans [38•,64••] reduces sympathetic activity, but does not affect respiration or end tidal carbon dioxide, is consistent with the distinct connection between glomus cells and central regulatory pathways as proposed above. This is further upheld by the finding that dopamine infusion to reversibly inactivate the carotid body had no effect on femoral blood flow, conductance or mean 
arterial pressure either at rest or during exercise, but did reduce the ventilatory response to hypoxia in healthy normotensive subjects [63]. This being the case, one might predict seeing heterogeneity amongst glomus cells in response to stimulants. Recently, a sub-population of glomus cells was found responsive to $\mathrm{pH}$, but not hypoxia and vice versa [68]. Moreover, there appears to be clear demarcation of inputs to neurons within the nucleus tractus solitarii (NTS), the central site of termination of carotid chemoreceptor primary afferents. There are distinct convergence patterns on to NTS cells receiving carotid body inputs, some being excitatory and others inhibitory, from baroreceptors, cardiac receptors, and pulmonary receptors $[69,70]$. Based on the patterns of reflex response from these inputs, this was interpreted as indicating the functional role of individual NTS cells in circuits controlling sympathetic, parasympathetic, respiratory outputs, or higher brain functions. This idea was consistent with the unique projection patterns of different carotid body activated NTS neurons [20]. Based on this, we hypothesise that there will a distinct sub-population of glomus cells within the carotid body that drive sympathetic activity uniquely (Fig. 1). We are underway with the quest to determine what this driver is.

Trzebski and colleagues studied 20 subjects with mild hypertension versus age and sex-matched control subjects [36]. Ventilatory and blood pressure responses to hypoxia were greater in the hypertensive subjects. Interestingly, there was a significant correlation between the responses to hypoxia and hypercapnia in the normotensive subjects but not the hypertensive subjects, suggesting a predominant role for hypoxic stimulation of peripheral chemosensors in hypertension. This supports the importance of hypoxic preconditioning for chemosensitivity. However, we argue that while chemosensitivity may be present in many chronic hypertensive patients who "precondition for chemosensitivity", it remains unclear if this is coincidental or even dependent upon underlying tonic hyperactivity. In support of this, we have seen recently a hypotensive effect resulting from dopamine-mediated inhibition of the $\mathrm{CB}$, while hypoxic chemosensitivity was low, in a hypertensive patient (L Ratcliffe, P Sobotka, J Paton - unpublished observation). Thus, whether chemosensitization of some type is required to increase tonicity, or if chemosensitivity can be increased without changing tonicity, is an open question. However, given our pilot data, it appears that tonicity can be increased without apparent chemosensitivity, at least to hypoxia. Indeed, the physiologic consequences of chemosensitivity and tonic hyperactivity activity may prove to be different.

\section{Conclusions and future perspectives}

In discussing carotid body chemoreceptors, Ponte and Purves (1974) remarked: "their function of ensuring adequate oxygenation of the brain may be the most important one they possess" [71]. This must include maintenance of cerebral perfusion brought about by increasing systemic arterial pressure. However, an assessment of carotid body reflex control on cerebral circulation and any modulation of this in cardiovascular disease will become important to fully appreciate Ponte and Purves' proposal.

With direct input to the caudal commissural nucleus tractus solitarii, the carotid body provides excitatory synaptic drive to neurons that is both powerful and long lasting relative to other inputs [20]. Carotid chemoreceptors generate sympathetic activity reflexively by activating rostral ventrolateral medullary pre-sympathetic neurons [72], and indirectly by exciting respiratory neurons connected to these sympathetic neurons [43•]. Importantly, carotid chemoreceptors are essential for inducing chronic potentiation of sympathetic activity and hypertension seen after intermittent hypoxia in rats, for example [42]. Thus, their activity can cause long-term synaptic plasticity within brainstem sympatho-excitatory networks. Recent work indicates that this may include alteration in the excitability of the respiratory neurons coupled to pre-motor sympathetic neurons (Moraes, DJA, Machado BH and Paton JFR - unpublished data). Future studies now need to assess how repeated carotid body activation triggers neuronal plasticity, and why this is to functionally specific neuronal types.

Future studies will need to determine the molecular basis for the differences in carotid body reflex sensitivity versus tonicity of chemoreceptor afferents, and whether these changes occur at the level of the glomus cell and/or on the terminal ending of the primary afferents. Since in hypertension (but also heart failure), there is an increase in carotid body reflex sensitivity such that evoked increases in sympathetic activity are larger in hypertensive animals [40 • ] and humans [35, 36], and since this occurs before the onset of hypertension $[40 \bullet \cdot$, a transcriptomic analysis may be optimal in the first instance.

Acknowledgments The financial support of the British Heart Foundation to Julian F.R. Paton is appreciated. Laura Ratcliffe is supported by Cibiem. Dagmara Hering, Jacek Wolf, and Krzysztof Narkiewicz are supported by the Foundation for Polish Science TEAM/2008-2/5 grant.

\section{Compliance with Ethics Guidelines}

Conflict of Interest Julian F.R. Paton has served as a consultant to Cibiem.

Laura Ratcliffe has received funding from Coridea for postgraduate studentship at University of Bristol.

Dagmara Hering has received research support from Cibiem.

Jacek Wolf has received research support from Cibiem.

Paul A. Sobotka is employed by Cibiem; has served as a consultant to Medtronic, Rox, and Ardelyx; has received payment for lectures, including service on speakers bureaus from Medtronic; has received payments for patents from Cibiem; has received royalties from 
Medtronic; and has received stock/stock options from Ardelyx and Cibiem.

Krzysztof Narkiewicz has received research support from Cibiem.

Human and Animal Rights and Informed Consent With regard to the authors' research cited in this paper, all procedures followed were in accordance with the ethical standards of the responsible committee on human experimentation (institutional and national) and with the Helsinki Declaration of 1975, as revised in 2008. Informed consent was obtained from all patients for being included in the studies.

In addition, all institutional and national guidelines for the care and use of laboratory animals were followed.

Open Access This article is distributed under the terms of the Creative Commons Attribution License which permits any use, distribution, and reproduction in any medium, provided the original author(s) and the source are credited.

\section{References}

Papers of particular interest, published recently, have been highlighted as:

- Of importance

-• Of major importance

1. Lohmeier TE, Iliescu R. Chronic lowering of blood pressure by carotid baroreflex activation: mechanisms and potential for hypertension therapy. Hypertension. 2011;57:880-6.

2. Heusser K, Tank J, Engeli S, et al. Carotid baroreceptor stimulation, sympathetic activity, baroreflex function, and blood pressure in hypertensive patients. Hypertension. 2010;55:619-26.

3. - Krum H, Schlaich M, Whitbourn R, et al. Catheter-based renal sympathetic denervation for resistant hypertension: a multicentre safety and proof-of-principle cohort study. Lancet. 2009;373(9671):127581. Landmark study that translated the idea of renal denervation performed in hypertensive rats in the 1980s for the treatment of human drug resistant hypertension. The study reports that the majority of patients responded (after 3 months) exhibiting a reduction of systolic blood pressure of XX $\mathrm{mmHg}$ and $\mathrm{ZZ} \mathrm{mmHg}$ by 6 months.

4. Carey RM. Resistant hypertension. Hypertension. 2013;61:74650.

5. Kearney PM, Whelton M, Reynolds K, et al. Global burden of hypertension: analysis of worldwide data. Lancet. 2005;365:21723

6. A scientific statement from the American Heart Association professional education committee of the council for high blood pressure research. Resistant hypertension: diagnosis, evaluation, and treatment. Circulation. 2008;117:e510-26.

7. Esler M, Kaye D. Sympathetic nervous system activation in essential hypertension, cardiac failure and psychosomatic heart disease. J Cardiovasc Pharmacol. 2000;35(7 Suppl 4):S1-7.

8. Grassi G. Sympathetic and baroreflex function in hypertension: implications for current and new drugs. Curr Pharm Des. 2004;10:357989.

9. Grassi G. Counteracting the sympathetic nervous system in essential hypertension. Curr Opin Nephrol Hypertens. 2004;13:513-9.

10. - Simms AE, Paton JF, Pickering AE, et al. Amplified respiratorysympathetic coupling in the spontaneously hypertensive rat: does it contribute to hypertension? J Physiol. 2009;587:597-610. First study to show that sympathetic activity is heightened in the spontaneously hypertensive rat before it develops hypertension, and that this was due to its elevated respiratory modulation. Importantly, the study demonstrated the contribution of this elevated respiratory-sympathetic modulation to vascular resistance and arterial pressure.

11. Smith PA, Graham LN, Mackintosh AF, et al. Sympathetic neural mechanisms in white-coat hypertension. J Am Coll Cardiol. 2002;40:126-32.

12. Smith PA, Graham LN, Mackintosh AF, et al. Relationship between central sympathetic activity and stages of human hypertension. Am J Hypertens. 2004;17:217-22.

13. Grimson KS, Orgain ES, Anderson B, et al. Total thoracic and partial to total lumbar sympathectomy, splanchnicectomy and celiac ganglionectomy for hypertension. Ann Surg. 1953;138:532-47.

14. Smithwick RH. Splanchnicectomy for essential hypertension; results in 1,266 cases. J Am Med Assoc. 1953;152:1501-4.

15. Malinow SH. Comparison of guanadrel and guanethidine efficacy and side effects. Clin Ther. 1983;5:284-9.

16. Esler MD, Krum H, Sobotka PA, Schlaich MP, Schmieder RE, Böhm M, et al. Renal sympathetic denervation in patients with treatment-resistant hypertension (The Symplicity HTN-2 Trial): a randomised controlled trial. Lancet. 2010;376:1903-9.

17. • Foss JD, Fink GD, Osborn JW. Reversal of genetic salt-sensitive hypertension by targeted sympathetic ablation. Hypertension. 2013;61:806-11. The importance of sympathetic inputs to the kidney and splanchnic vascular bed in the generation of neurogenic hypertension were demonstrated. In Dahl salt-sensitive rats on high salt, the evoked hypertension was lowered after the targeted sympathectomy. The additive effect of combined sympathectomy indicates distinct mechanisms are involved.

18. Donoghue S, Felder RB, Jordan D, et al. The central projections of carotid baroreceptors and chemoreceptors in the cat: a neurophysiological study. J Physiol. 1984;347:397-409.

19. Marshall JM. Peripheral chemoreceptors and cardiovascular regulation. Physiol Rev. 1994;74:543-94.

20. Paton JFR, Li Y-W, Kasparov S. Reflex response and convergence of pharyngoesophageal and peripheral chemo- receptors in the nucleus of the solitary tract. Neuroscience. 1999;93:143-54.

21. Barnett S, Mulligan E, Wagerle LC, et al. Measurement of carotid body blood flow in cats by use of radioactive microspheres. J Appl Physiol. 1988;65:2484-9.

22. Fitzgerald RS, Eyzaguirre C, Zapata P. Fifty years of progress in carotid body physiology-invited article. Adv Exp Med Biol. 2009;648:19-28.

23. de Burgh Daly M. Peripheral arterial chemoreceptors and respiratory-cardiovascular integration. Monograph. Oxford: Clarendon Press; 1997.

24. Stulbarg MS, Winn WR, Kellett LE. Bilateral carotid body resection for the relief of dyspnea in severe chronic obstructive pulmonary disease. Physiologic and clinical observations in three patients. Chest. 1989;95:1123-8.

25. Hilton SM, Marshall JM. The pattern of cardiovascular response to carotid chemoreceptor stimulation in the cat. J Physiol. 1982;326:495513.

26. Angel A, Harris MC. The effect of chemoreceptor stimulation on the centripetal transfer of somatosensory information in the urethane-anaesthetized rat. Neuroscience. 1998;86:321-35.

27. De Burgh Daly M, Scott MJ. An analysis of the primary cardiovascular reflex effects of stimulation of the carotid body chemoreceptors in the dog. J Physiol. 1962;162:555-73.

28. Daly MD, Scott MJ. The cardiovascular responses to stimulation of the carotid body chemoreceptors in the dog. J Physiol. 1963;165:179-97.

29. Paton JFR, Boscan P, Pickering AE, et al. The yin and yang of cardiac autonomic control: vago-sympathetic interactions revisited. Brain Res Rev. 2005;49:555-65.

30. Somers VK, Mark AL, Abboud FM. Interaction of baroreceptor and chemoreceptor reflex control of sympathetic nerve activity in normal humans. J Clin Invest. 1991;87:1953-7. 
31. Marshall JM. Interaction between the responses to stimulation of peripheral chemoreceptors and baroreceptors: the importance of chemoreceptor activation of the defence areas. J Auton Nerv Syst. 1981;3:389-400.

32. Spyer KM. Neural mechanisms involved in cardiovascular control during affective behaviour. Trends Neurosci. 1989;12:506-13.

33. Przybylski J, Trzebski A, Czyzewski T, et al. Responses to hyperoxia, hypoxia, hypercapnia and almitrine in spontaneously hypertensive rats. Bull Eur Physiopathol Respir Clin Respir Physiol. 1982;18:145-54.

34. Trzebski A. Arterial chemoreceptor reflex and hypertension. Hypertension. 1992;19:562-6.

35. Somers VK, Mark AL, Abboud FM. Potentiation of sympathetic nerve responses to hypoxia in borderline hypertensive subjects. Hypertension. 1988;11:608-12.

36. Trzebski A, Tafil M, Zoltowski M. Increased sensitivity of the arterial chemoreceptor drive in young men with mild hypertension. Cardiovasc Res. 1982;16:163-72.

37. Tafil-Klawe M, Trzebski A, Klawe J, et al. Augmented chemoreceptor reflex tonic drive in early human hypertension and in normotensive subjects with family background of hypertension. Acta Physiol Pol. 1985;36:51-8.

38. - Sinski M, Lewandowski J, Przybylski J. Tonic activity of carotid body chemoreceptors contributes to the increased sympathetic drive in essential hypertension. Hypertens Res. 2012;35:487-91. This study reveals an oxygen sensitive tonically active excitatory drive from the carotid body in humans with hypertension. The authors show reductions in arterial pressure and muscle sympathetic nerve activity when hypertensive patients are given $100 \%$ oxygen to breathe. The authors conclude that there is a major role for the carotid body in human essential hypertension.

39. Izdebska E, Cybulska I, Sawicki I. Postexercise decrease in arterial blood pressure, total peripheral resistance and in circulatory responses to brief hyperoxia in subjects with mild essential hypertension. J Hum Hypertens. 1998;12:855-60.

40. •• Tan ZY, Lu Y, Whiteis CA, et al. Chemoreceptor hypersensitivity, sympathetic excitation, and overexpression of ASIC and TASK channels before the onset of hypertension in SHR. Circ Res. 2010;106:536-45. This study reports the relative roles of acid sensitive and sodium and potassium channels in the detection of low $\mathrm{pH}$. There was a significant increase in the depolarizing effect of low pH in glomus cells from spontaneously hypertensive rats versus normotensive rats, which was caused by overexpression of 2 acid-sensing non-voltage-gated channels. It also shows that these channels were over expressed prior to the onset of hypertension in spontaneously hypertensive rats and that these immature animals have heightened chemosensitivity, as revealed by greater sympatho-excitatory responses relative to age-matched and sexmatched normotensive rats.

41. Ponikowski P, Chua TP, Anker SD, et al. Peripheral chemoreceptor hypersensitivity: an ominous sign in patients with chronic heart failure. Circulation. 2001;104:544-9.

42. Fletcher EC, Lesske J, Behm R, et al. Carotid chemoreceptors, systemic blood pressure, and chronic episodic hypoxia mimicking sleep apnea. J Appl Physiol. 1992;72:1978-84.

43. - Zoccal DB, Simms AE, Bonagamba LG, et al. Increased sympathetic outflow in juvenile rats submitted to chronic intermittent hypoxia correlates with enhanced expiratory activity. J Physiol. 2008;586:3253-65. This is the first study to show that the hypertension evoked by chronic intermittent hypoxia is generated, in part, by elevated respiratory modulation of sympathetic activity. This was shown to be due to: (i) enhanced magnitude of the coupling seen in control rats and (ii) a novel respiratory related sympathetic burst of activity generated late in the expiratory interval. The latter sympathetic burst was coupled with expiratory abdominal motor activity, suggesting that chronic intermittent hypoxia also caused active expiration.
44. Kara T, Narkiewicz K, Somers VK. Chemoreflexes - physiology and clinical implications. Acta Physiol Scand. 2003;177:37784.

45. Narkiewicz K, Somers VK. Sympathetic nerve activity in obstructive sleep apnoea. Acta Physiol Scand. 2003;177:385-90.

46. •- Narkiewicz K, van de Borne PJH, Montano N, et al. Contribution of tonic chemoreflex activation to sympathetic activity and blood pressure in patients with obstructive sleep apnea. Circulation. 1998;97:943-5. Using $100 \%$ oxygen to inactivate the carotid bodies, this study showed that patients with sleep apnea have highly active carotid bodies that participate in the generation of their high blood pressure and high sympathetic nerve activity.

47. Dampney RAL, Tagawa T, Horiuchi J, Potts PD, Fontes M, Polson JW. What drives the tonic activity of presympathetic neurons in the rostral ventrolateral medulla? Clin Exp Pharmacol Physiol. 2000;27:1049-53.

48. Heath D, Smith P, Fitch R, et al. Comparative pathology of the enlarged carotid body. J Comp Pathol. 1985;95:259-71.

49. Clarke JA, de Burgh Daly M, Ead HW. Vascular analysis of the carotid body in the spontaneously hypertensive rat. In: Data PG, editor. Neurobiology and cell physiology of chemoreception. New York: Plenum Press; 1993. p. 3-8.

50. Prabhakar NR, Semenza GL. Gaseous messengers in oxygen sensing. J Mol Med (Berl). 2012;90:265-72.

51. Schultz HD. Angiotensin and carotid body chemoreception in heart failure. Curr Opin Pharmacol. 2011;11:144-9.

52. Li YL, Sun SY, Overholt JL, et al. Attenuated outward potassium currents in carotid body glomus cells of heart failure rabbit: involvement of nitric oxide. J Physiol. 2004;555:219-29.

53. - Ding Y, Li YL, Schultz HD. Role of blood flow in carotid body chemoreflex function in heart failure. J Physiol. 2011;589:245-58. Using microspheres in rabbits, blood flow to the carotid body was modulated by carotid artery occlusion. By reducing carotid body blood flow, chemoreceptor reflex sensitivity and tonicity was elevated and associated with a decrease in neuronal nitric oxide synthase, increased angiotensin type 1 receptor expression and angiotensin II concentration, as well as decreased outward potassium current. These changes were consistent with those found in rabbits with heart failure, supporting the notion that reduction of carotid body perfusion may itself heighten carotid body signaling.

54. Wyatt CN, Pearson SA, Kumar P, et al. Key roles for AMPactivated protein kinase in the function of the carotid body? Adv Exp Med Biol. 2008;605:63-8.

55. Lam SY, Liu Y, Ng KM, et al. Chronic intermittent hypoxia induces local inflammation of the rat carotid body via functional upregulation of proinflammatory cytokine pathways. Histochem Cell Biol. 2011;137:303-17.

56. - Kato K, Wakai J, Matsuda H, et al. Increased total volume and dopamine $\beta$-hydroxylase immunoreactivity of carotid body in spontaneously hypertensive rats. Auton Neurosci Basic Clin. 2012;169:49-55. This paper shows that spontaneously hypertensive rats have an enlarged carotid body and enhanced immunoreactivity for dopamine b-hydroxylase. The authors propose that the morphology of the carotid body may be affected by the sympathetic nervous system, and that carotid body signal transduction is regulated by NA in glomus cells of hypertensive rats.

57. De Caro R, Macchi V, Sfriso MM, et al. Structural and neurochemical changes in the maturation of the carotid body. Respir Physiol Neurobiol. 2013;185:9-19.

58. Marvar PJ, Lob H, Vinh A, et al. The central nervous system and inflammation in hypertension. Curr Opin Pharmacol. 2011;11:15661.

59. Fisher JP, Paton JFR. The sympathetic nervous system and blood pressure in humans: implications for hypertension. J Hum Hypertens. 2012;26:463-75. 
60. Zubcevic J, Waki H, Raizada MK, et al. Autonomic-immunevascular interaction: an emerging concept for neurogenic hypertension. Hypertension. 2011;57:1026-33.

61. - Ackland GL, Kazymov V, Marina N, et al. Peripheral neural detection of danger-associated and pathogen-associated molecular patterns. Crit Care Med. 2013;[Epub ahead of print]. Data are presented that the carotid body glomus cells act to detect pathogen and danger associated molecules. Toll-like receptor activation resulted in inflammasome-dependent mechanism within glomus cells similar to that described in immune cells. The result of this was $I L-1 b$ release, which acted in an autocrine manner to raise carotid body activity.

62. Paton JFR, Sobotka PA, Fudim M, et al. The carotid body as a therapeutic target for treatment of sympathetically mediated diseases. Hypertension. 2013;61:5-13.

63. Stickland MK, Fuhr DP, Haykowsky MJ, et al. Carotid chemoreceptor modulation of blood flow during exercise in healthy humans. J Physiol. 2011;589:6219-30.

64. •- McBryde FD, Abdala AP, Hendy EB, et al. A novel and translatable approach for the treatment of neurogenic hypertension. Nat Commun. 2013;(in revision). This study demonstrates the mechanisms underpinning the hypotensive response to carotid body ablation in spontaneously hypertensive rats. These include substantial reductions in renal sympathetic activity, improved baroreceptor reflex gain, improved renal function and reduced tissue inflammation. The study also shows that there is a summative interaction between renal denervation and carotid body ablation.

65. Abdala AP, McBryde FD, Marina N, et al. Hypertension is critically dependent on the carotid body input in the spontaneously hypertensive rat. J Physiol. 2012;590:4269-77.

66. Nakayama K. Surgical removal of the carotid body for bronchial asthma. Dis Chest. 1961;40:595-604.

67. Winter B, Whipp BJ. Immediate effects of bilateral carotid body resection on total respiratory resistance and compliance in humans. Adv Exp Med Biol. 2004;551:15-21.

68. Lu Y, Whiteis CA, Sluka KA, et al. Responses of glomus cells to hypoxia and acidosis are uncoupled, reciprocal and linked to ASIC3 expression: selectivity of chemosensory transduction. J Physiol. 2013;591:919-32.

69. Paton JFR. Convergence properties of solitary tract neurones driven synaptically by cardiac vagal afferents in the mouse. J Physiol. 1998;508:237-52.

70. Paton JFR. Pattern of cardiorespiratory afferent convergence to solitary tract neurons driven by pulmonary vagal C-fiber stimulation in the mouse. J Neurophysiol. 1998;79:2365-73.

71. Ponte J, Purves MJ. The role of the carotid body chemoreceptors and carotid sinus baroreceptors in the control of cerebral blood vessels. J Physiol. 1974;237:315-40.

72. Koshiya N, Huangfu D, Guyenet PG. Ventrolateral medulla and sympathetic chemoreflex in the rat. Brain Res. 1993;609:17484. 